resulting from a direct aortic injury caused by a fractured wire by means of emergency surgical intervention.

\section{References}

1. Cope SA, Rodda J. Cardiac tamponade presenting to the emergency department after sternal wire disruption. Emerg Med J. 2004;21:389-90.

2. Hazelrigg SR, Staller B. Migration of sternal wire into ascending aorta. Ann Thorac Surg. 1994;57:1023-4.
3. Freund E, Nachman R, Gips H, Hiss J. Migration of a Kirschner wire used in the fixation of a subcapital humeral fracture, causing cardiac tamponade: case report and review of literature. Am J Forensic Med Pathol. 2007;28: 155-6.

4. Lyons FA, Rockwood CA Jr. Migration of pins used in operations on the shoulder. J Bone Joint Surg. 1990;72:1262-7.

5. Schechter DC, Gilbert L. Injuries of the heart and great vessels due to pins and needles. Thorax. 1969;24:246-53.

\title{
Mediastinoscopy-assisted ligation of an aberrant right subclavian artery through a supraclavicular approach
}

\author{
Narain Moorjani, MD, FRCS, ${ }^{\mathrm{a}}$ Nancy Mohsen, MD, ${ }^{\mathrm{b}}$ and Percy Boateng, MD, ${ }^{\mathrm{a}}$ Philadelphia, Pa
}

Video clip is available online.

An aberrant right subclavian artery occurs in approximately $0.5 \%$ of the population. ${ }^{1}$ Compression of the esophagus and trachea might result from a retromediastinal course of the aberrant artery. Surgical treatment in symptomatic patients usually involves ligation of the artery close to its origin from the aortic arch followed by right common carotidsubclavian artery bypass. This report describes the use of a cervical mediastinoscope through a right supraclavicular incision to aid access and visualization of the aberrant right subclavian artery at its origin from the distal aortic arch, without the need for a left thoracotomy.

\section{CLINICAL SUMMARY}

A 28-year-old woman presented with a 2-month history of progressive dysphagia to solids. An esophagram demonstrated indentation of the upper esophagus with transient delay of contrast, which is consistent with extrinsic compression (Figure 1, A). Subsequent contrast-enhanced computed tomographic analysis revealed an aberrant right subclavian artery originating from the aortic arch, distal to

From the Department of Cardiothoracic Surgery ${ }^{a}$ and Radiology, ${ }^{b}$ Hahnemann University Hospital, Drexel University College of Medicine, Philadelphia, Pa. Disclosures: None.

Received for publication March 10, 2009; revisions received June 4, 2009; accepted for publication June 28, 2009; available ahead of print Aug 26, 2009.

Address for reprints: Narain Moorjani, MD, FRCS, Department of Cardiothoracic Surgery, Hahnemann University Hospital, Drexel University College of Medicine, Philadelphia, PA 19103 (E-mail: narain.moorjani@doctors.org.uk).

J Thorac Cardiovasc Surg 2010;140:e20-1

$0022-5223 / \$ 36.00$

Copyright (c) 2010 by The American Association for Thoracic Surgery doi:10.1016/j.jtcvs.2009.06.026 the left subclavian artery, and passing posterior to the esophagus (Figure 1, B, and Video 1). The origin of the aberrant artery measured $1.1 \mathrm{~cm}$, with no aneurysmal dilatation or Kommerell's diverticulum. A common origin of the left and right common carotid arteries (truncus bicaroticus) from the aortic arch was also observed (Figure 1, B).

In view of her symptoms, surgical treatment was performed by using a 6-cm right supraclavicular incision and division of the clavicular head of the sternocleidomastoid muscle. After mobilization of the right internal jugular vein, the right common carotid artery was identified in the carotid sheath while protecting the vagus nerve. The aberrant right subclavian artery was identified posteriorly, coursing behind the esophagus and right recurrent laryngeal nerve. Because access to the origin of the aberrant right subclavian artery was limited, a standard cervical mediastinoscope was used to aid visualization of the proximal part of the artery (Figure 1, C). By using blunt dissection through the mediastinoscope, the artery was mobilized free from the esophagus (with an orogastric tube in situ) down to its origin from the aortic arch. After systemic heparinization, the right subclavian artery was ligated and oversewn at its origin. Intraoperative Doppler scanning and direct visualization through the mediastinoscope ensured that the ligation was proximal to the vertebral artery. The distal free end of the right subclavian artery was then sutured to the right common carotid artery by using an end-to-side anastomosis (Figure 1, $D)$. Intraoperative Doppler examination of the right common carotid, subclavian, and vertebral arteries demonstrated excellent flows. The patient was discharged on the fourth postoperative day, with normal swallowing, vocal cord function, neurological status, and perfusion of the right upper limb. Postoperative computed tomographic analysis demonstrated the right subclavian-common carotid artery bypass and the ligated aberrant subclavian artery on the distal aortic arch (Figure 1, $E$ and $F$, and Video 2). 

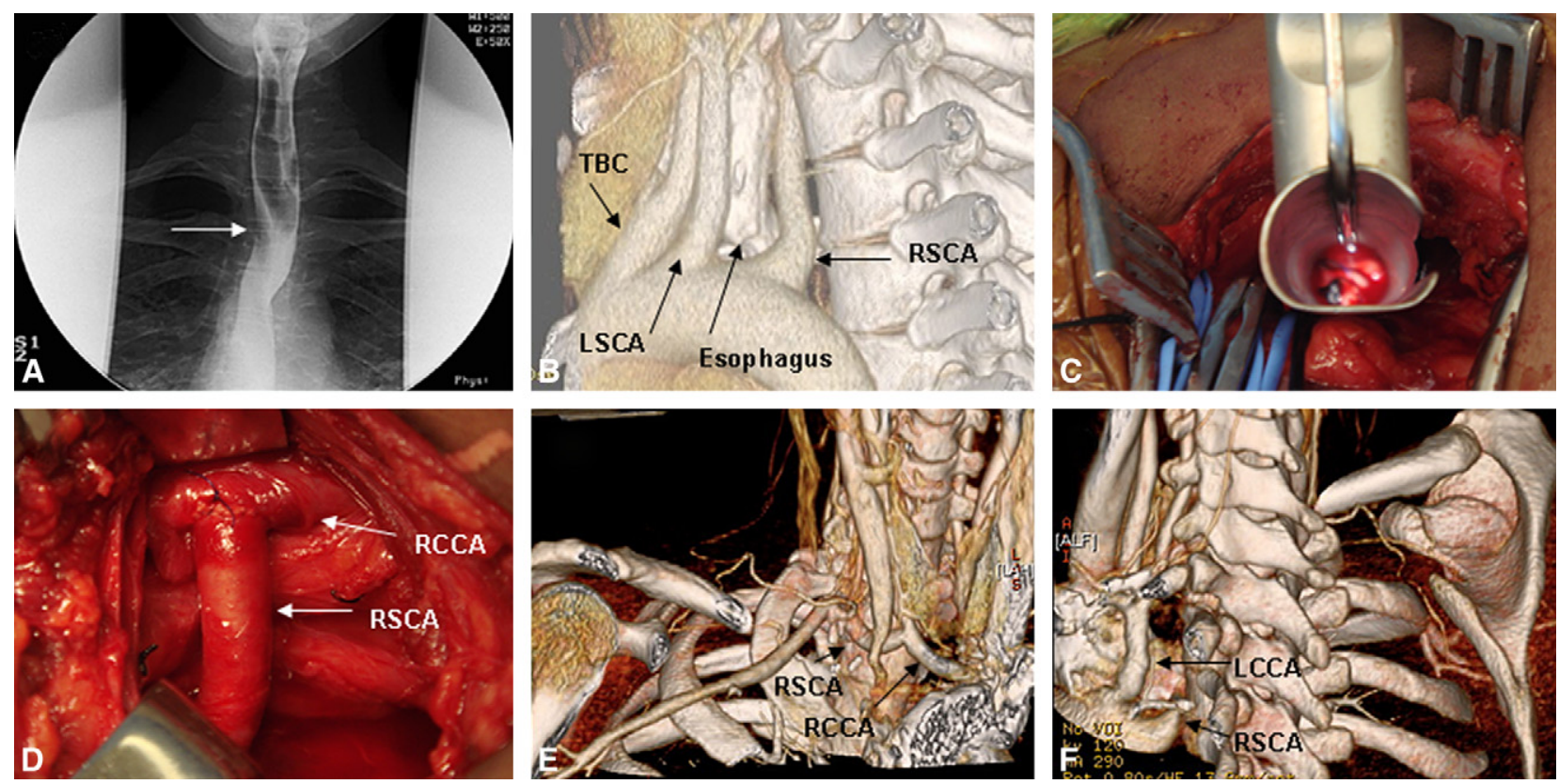

FIGURE 1. A, Preoperative double-contrast barium esophagram demonstrating extrinsic esophageal compression (arrow). B, Preoperative 3-dimensional computed tomographic reconstruction demonstrating an aberrant right subclavian artery (RSCA; arrow) originating distal to the left subclavian artery ( $L S C A)$, with a retromediastinal course causing esophageal compression. A common origin of the left and right common carotid arteries (truncus bicaroticus [TBC]) from the aortic arch is also observed. $\mathrm{C}$ and $\mathrm{D}$, Operative images demonstrating the use of a mediastinoscope to aid visualization and lighting to gain access to the proximal aberrant right subclavian artery at its origin from the aortic arch $(\mathrm{C})$ and completed right subclavian (RSCA)-right common carotid artery (RCCA) bypass (D). E and F, Postoperative 3-dimensional computed tomographic reconstruction demonstrating patent right subclavian-common carotid artery bypass (E) and a ligated aberrant subclavian artery on the distal aortic arch (F).

\section{DISCUSSION}

An aberrant right subclavian artery is the most common congenital abnormality of the aortic arch. The proximal part of the right subclavian artery normally originates from the fourth right aortic arch. Abnormal involution of this arch during embryologic development and persistence of the distal right dorsal aorta results in an aberrant origin of the right subclavian artery distal to the left subclavian artery. From its aberrant origin, the artery usually crosses the midline between the esophagus and the thoracic vertebrae. Although the majority of patients with aberrant right subclavian arteries are asymptomatic, esophageal compression can result, causing difficulty in swallowing. Dysphagia lusoria was first described in 1794 by Bayford, who associated the postmortem diagnosis of aberrant right subclavian artery with the patient's dysphagia symptoms. ${ }^{2}$

Surgical intervention is usually performed in patients when the dysphagia becomes persistent. Surgical approaches include left thoracotomy, right supraclavicular incision, the combined left thoracotomy and right supraclavicular approach, or right thoracotomy. ${ }^{3}$ Hybrid procedures, including percutaneous endovascular occlusion of the aberrant artery followed by right common carotid-subclavian bypass through a right supraclavicular incision, have also been described. ${ }^{4}$ Although previous reports of 1-stage ligation of the aberrant artery and common carotid-subclavian bypass through a right supraclavicular approach have been described, ${ }^{3}$ the use of a mediastinoscope as an adjunct to surgical intervention has not been previously reported. As opposed to the direct approach, the improved lighting and visualization allows greater access of the aberrant right subclavian artery at its origin from the distal aortic arch, reducing the size of any residual stump and allowing better access to any bleeding that is encountered. In cases of Kommerell's diverticulum, however, this less invasive method might not be possible because greater access to the distal aortic arch might be required.

In conclusion, this report describes the operative management of a patient with an aberrant right subclavian artery presenting with dysphagia lusoria. The use of a mediastinoscope to aid visualization of the proximal artery at its origin should be considered when a single-stage right supraclavicular approach is used.

\section{References}

1. Kieffer E, Bahnini A, Koskas F. Aberrant subclavian artery: surgical treatment in thirty-three adult patients. J Vasc Surg. 1994;19:100-11.

2. Asherson N. David Bayford. His syndrome and sign of dysphagia lusoria. Ann R Coll Surg Engl. 1979;61:63-7.

3. Carrizo GJ, Marjani MA. Dysphagia lusoria caused by an aberrant right subclavian artery. Tex Heart Inst J. 2004;31:168-71.

4. Shennib H, Diethrich EB. Novel approaches for the treatment of the aberrant righ subclavian artery and its aneurysms. J Vasc Surg. 2008;47:1066-70. 